\title{
IIII WAVENUMBERS FOR CURRENTS ON INFINITE- AND F'INITE-I.ENGTII WIRES IN A CHIRAI, MEDIUM
}

\author{
Authoms: A. K. Bhattacharyya, PSI, \\ G. J. Burke, LI,NI, \\ H. K. Miller, MFF-3
}

\author{
illumill" IO IFEL-APS International Symposium \\ Chicago, II. \\ JuIY IH-25, 109?
}

\section{IDSC'I.AIMF.K}

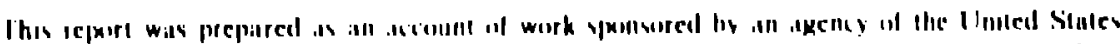

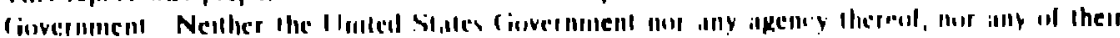

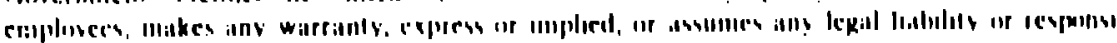

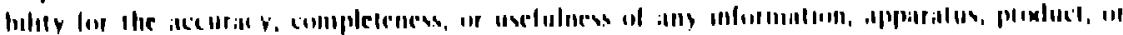

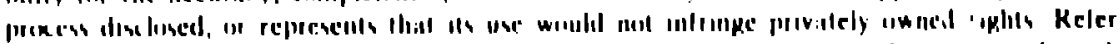

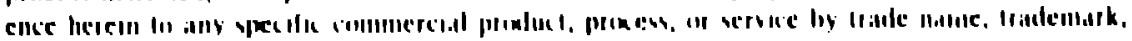

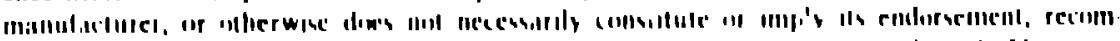

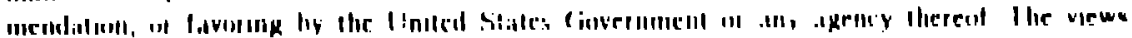

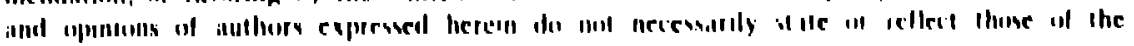

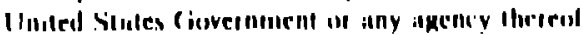

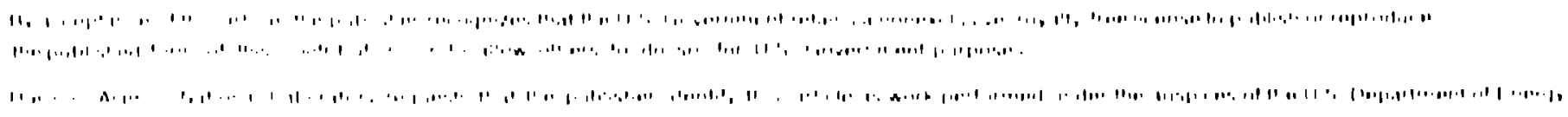




\title{
WAVENUMBERS FOR CURRENTS ON INFINITE- AND FINITE-LENGTH WIRES IN A CHIRAL MEDIUM
}

\author{
A. K. Bhattacharyya, PSL \\ New Mexico State University \\ PO Box 3002 \\ Las Cruces, NM 88003-0002
}

\author{
G. J. Burke, L-156 \\ Lawrence Livermore National Laboratory \\ PO Box 808 \\ Livermore, CA 9455()
}

\author{
E. K. Miller, MS J580 \\ Los Alamos National Laboratory \\ PO Box 1663 \\ Los Alamos, NM 87545
}

\section{INTRODUCTION}

There is incicasing interest in determining the electromagnetic properics of matcrial media differing from free space and the effects therenf on the radiation, propagation, and scaucring of electromagnetic ficlds. A malerial propeity of special present interest is that of chirality. Chirality manifests itself as a "handedness" wherein a chiral medium does not support propagation of a linearly-polarized plane waye, but which instead decomposes into two circularly-polarized waves that propagatc at different speeds. Initial work in this area was devoted to developing various analytical solutions to some hasic preblems such as the Green's Dyadic for a point current source [Basiri el al. (1986)]. Altention is now being increasingly devoled to using this carly work for a varicty of applications such as analyzing antennas in chiral media (Jageard ct. al. (1991)); scaucring from chiral objects [Bhallacharyya (1991); scaltering from objects having chiral coalings [Uslenghi (1990)]; and reflection from planar chiral interfaces [Lakhlakia ct. al. (1986)].

The freus of the work described here is decermining the wavenumberis $(=-\alpha-j \beta)$ of the current waves excited on wire antennas located in an infinite chiral medium using two complementiary approaches. One is wo use an extension of an existing compuler model (NEC) |Burke and Poggio, (1981)] that permits modeling oi arbitrary wire objecks locuted in un infinitc chiral medium. The outcr is to develop a solution for an infinitely long cylindrical antetina also localed in an infinite chiral medium. The taller canonical problem. is of interest in its own right as well as nroviding a means for achic ving mulual validav's with the NEC model.

\section{EINITE-LENGTH WIRES IN A CIIKAL_MEDUUM}

The thin-wirc, electric-field integral equation for a perfoctly-conducting, wire can be exp,ressed as

$$
\mathbf{s} \cdot \mathbf{E}^{\mathrm{inc}}(\mathbf{r})=-j \omega \mu \int_{\mathrm{C}(\mathbf{r})} \mathbf{s} \cdot\left|I\left(\mathbf{r}^{\prime}\right) \cdot \Gamma\left(\mathbf{r}, \mathbf{r}^{\prime}\right) \mathrm{d}\right| \mathbf{r}^{\prime} \mid ; \mathbf{r} \in \mathbf{C}(\mathbf{r})
$$

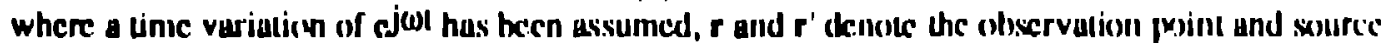
puint where the unit ungenl vextors are given by and s', respectively, Finc'( $r)$ is ule ' incident" ficld exciting the wire, $C^{\prime}(r)$ descerilkes the spatial contour of the wire, and $r^{\prime}\left(r, r^{\prime}\right)$ is the (irecris

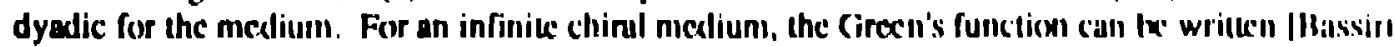
cl. ul. $(19 \times(1))$

$$
\begin{gathered}
\Gamma_{\text {diml } 1}\left(r, r^{\prime}\right)=a_{1}\left(u+\frac{1}{h_{1}} u \times \nabla+\frac{1}{h_{1}^{2}} \nabla \nabla\right) \frac{e^{-j h_{1} k}}{4 \pi k}+a_{2}\left(u-\frac{1}{h_{2}} u \nabla+\frac{1}{h_{2}^{2}} \nabla \nabla\right) \frac{e^{j h h_{2} k}}{4 \pi k} \\
a_{1}=\frac{h_{1}^{2}-k^{2}}{h_{1}^{2}-h_{2}^{2}}, a_{2}=-\frac{h_{2}^{2}-k^{2}}{h_{1}^{2}-h_{2}^{2}}
\end{gathered}
$$

AP.S 1992, Chiral Medium, Page I 


$$
h_{1,2}= \pm \omega \mu \gamma+\sqrt{(\omega, \gamma)^{2}+k^{2}}
$$

where $u$ is the unit dyad and the constant $\gamma_{c} \cdot m$ mines the degree of medium chirality as expressed by the constitutive relationships [Basiri, eL al. '1986)]

$$
D=\epsilon E+j \gamma B \text { and : }=j \gamma E+B / \mu \text {. }
$$

The wavenumber $k=\omega \sqrt{\varepsilon \mu}$ is the achiral-met $\mathrm{a}$ wavenumber and $\mathrm{c}$ and $\mu$ are the meflum permituvity and permeability respectively. As can be seen, the proportionality quantities al and a2 depend on the wavenumbers $h_{1}$ and $h_{2}$ for the two circularly-polarized waves that propagate in the chiral medium.

The NEC computer model has been modified to include the chiral Gruen's function to permit the modeling of arbitrary wire objects exciled as antennas and scaluerers in a chiral medium for which some initiai results have been reported elsewherc [Bhaltacharyya, Burke and Miller (1992a), (1992b)]. Extending NEC to the problem of a chiral medium somewhat complicales computing the electric fields of a general wire object because it is no longer possible to obtain the ficlds of tice sine and cosine terms of the three-term current basis used in NEC in closed form because of the two different propagation constants. For example, the z-component of the field has the form

$$
\begin{gathered}
E_{2}(\rho, z)=\frac{-j \eta l_{0}}{4 \pi k_{1}}\left\{\left(\begin{array}{c}
\sin k_{s^{\prime}} z^{\prime} \\
\cos k_{s} z^{\prime}
\end{array}\right)\left[\left(z-z^{\prime}\right)^{2} \frac{1+j k_{1} k}{R^{2}}-k_{s}\left(\begin{array}{c}
\cos k_{s} z^{\prime} \\
-\sin k_{s} z^{\prime}
\end{array}\right)\right] \frac{e^{-j k_{1} k}}{R}\right]_{-\delta}^{\delta} \\
\left.+\left(k_{1}^{2}-k_{s}^{2}\right) \int_{-\delta}^{\delta}\left(\begin{array}{c}
\sin k_{s} z^{\prime} \\
\cos k_{s} z^{\prime}
\end{array}\right) \frac{e^{-j k_{1} R}}{R} d z^{\prime}\right\}
\end{gathered}
$$

for the sine and cosine terms respectively and a current "segment" $2 \delta$ long lociled on the $z$-axis of a cylindrical coorcinate system. When the medium propagation cunstant $k_{1}$ cquals the basis propagation constant $\mathbf{k}_{\mathbf{s}}$, the $\mathbf{l e r m s}$ requiring numerical integration cuncel, and the ficld forms revert to those employed for an infinite, achiral, mediun. Good results are oblined from NEC using $k_{s}$ - Vh1 h2. Evgluating the field of the constant term of the current basis, which has no propagation constant for the current, is essentially unaffected ty the medium chirality except for the iact that a field components is produced for $h_{1}$ aidd $h_{2}$. The NEC estimates for the propagition constint are based on solving for the current on a wire six or so) wavelengths hong exciled a yuanter wavelengut from one end and having a resistive. load at the other end in reduce the current sunding-wave ratio. The compiex wavenumber was then obtuined from a least-syuares fit to the log of inagnilude and tix phuse of the current using five match points over each segment.

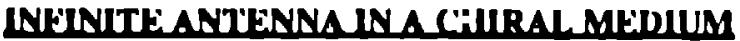

One starting point for modeling an infinite cylindrical ante'unu of rudius a lexulted on uke zaxis of a cylindrical cororlinate system is w writc an integral equation for the currellt as

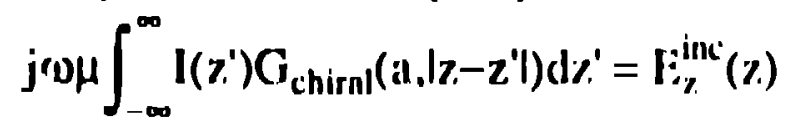

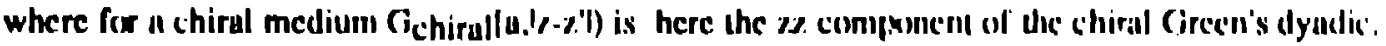
The solution wo this equation can he expressedl as a fourier integral of the current, i.e., IMiller $(1 \times, 7) \mid$

$$
I(x)=C \int_{-\infty}^{-\pi} e^{\| l \prime} i(\beta) d \mid \beta
$$

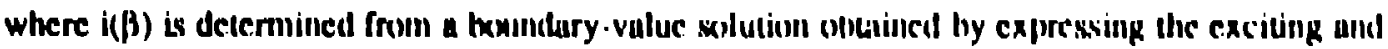

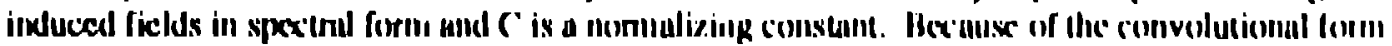

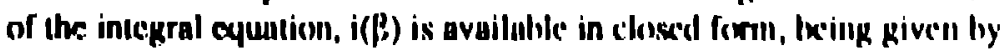




$$
i(\beta)=\frac{-j}{\eta} S(\beta) \frac{\left[\frac{a_{1} \lambda_{1}}{h_{1}} \frac{\partial}{\partial\left(\lambda_{1} a\right)} H_{0}^{(2)}\left(\lambda_{1} a\right)+\frac{a_{2} \lambda_{2}}{h_{2}} \frac{\partial}{\partial\left(\lambda_{2} a\right)} H_{0}^{(2)}\left(\lambda_{2} a\right)\right]}{\left[\frac{a_{1} \lambda_{1}^{2}}{h_{1}^{2}} H_{0}^{(2)}\left(\lambda_{1} a\right)+\frac{a_{2} \lambda_{2}^{2}}{h_{2}^{2}} H_{0}^{(2)}\left(\lambda_{2} a\right)\right]}
$$

where $S(\beta)$ is the spatial spectrum of the incident field, $H_{0}$ is a Hankel function of the serond kind, and $\lambda_{i}=\sqrt{h_{i}^{2}-\beta^{2}}$.

Evaluation of the infinite-a cnna current can be accomplished using numerical quadrature or analytical approximation of the dominant contributions to the integral. For our purposes, we do not need the explicit 2-dependent current, but can instcad determine the propagution constant from the poles oi the current spectrum to oblain the results which follow. We note that the denominator of $i(\beta)$ involves both chiral-medium waves which suggests that the mode associated with the denominalor zero might be described as a "hybrid" wave.

\section{NUMERICAL_RESULTS}

Wavenumber results for the current as obtained from the infinite-antenna model and from NEC arc shown in Fig. 1. The allenuation $(-\alpha)$ and propagation $(-j \beta)$ components of the wavenumber are compared respectively as a function of the chirality constant for two different values of wire radius. Good agreement is obtained between the NEC and infinite-antenna values over a chirality range of $10^{-4}$ to about 0.05 . For the vi.lues of chirality shown here, the chiral effect is seen to be greater with larger wire radii. The excellent agruement obtained confirms both the NEC and infinite-wire solutions.

The magniwde of the NEC current on the wire for various wire radii and a chirality constant $\gamma=$ 0.01 for a 30-wavelength (froc space) wire inodeled using 500 segments is shown in Fig. 2. It can be seen for the case investigated, that as wire radius is increased, there is a transition from a more rapidly-altenuated current near the source w a less-attenualed current further down the wire. The near-source current is a "fast-wave" mode where the propagation constunt is less than in the ambient medium which makes a uransition to an even "faster" mode which exhibits snialler altenuation, as shown in Fig. 3. The altenuation and propagation constants (normalized to ko tor the first region as oblained from NEC, $-0.12^{-} ;-j 0.9446$ match those computed fiom the polc of the current spectrum, -0.1280 -j0.9444, quite well. In the faster-wave regiott, the NEC propagation constant, -j0.4134, is elose to the value of h2 which is -j0.4142.

\section{REFGRENCES}

Bussiri, S., N. Enghelu and C. H. Papas (1986), Alla freque'nza, LV, 2, p1. 8.3-8.,

Bhallacharyya, A. K. (1960)), Elertronicrs Lellers, 26, 14, pp. IO(X)-I(K)7.

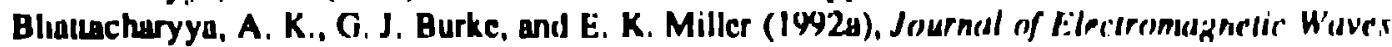
and Applicution.r, VNU Scieixe Press, accepted for puhlication.

Bhetlecharyya, A. K., G. J. Burke, and E. K. Miller (1992b), Microw'ave and (1)ptical Terchnollegy. Letiers, acceptad for publicution.

Burke, G. J. and A. J. Poggio (1981), "Numerical Electromagnelic Code (NI:C)--Method of

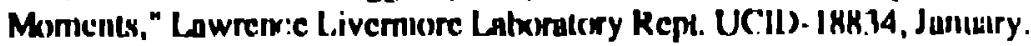

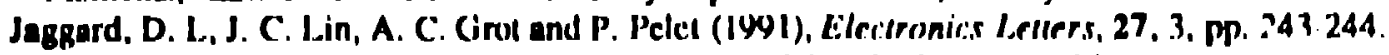

Lakhtakia, A., V. K. Varudun and V. V. Varudan (19)6), IE:EE Tran.r. E:ler:tromak. C'ompkat., EMC:28, 2.

Miller, E. K. (1964), Radion Srienice, 2, pp. 1431 14.35.

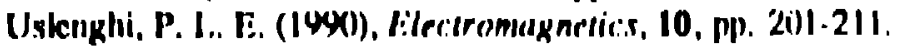

AP-S 1992, Chinal Mediun, Puge 3 

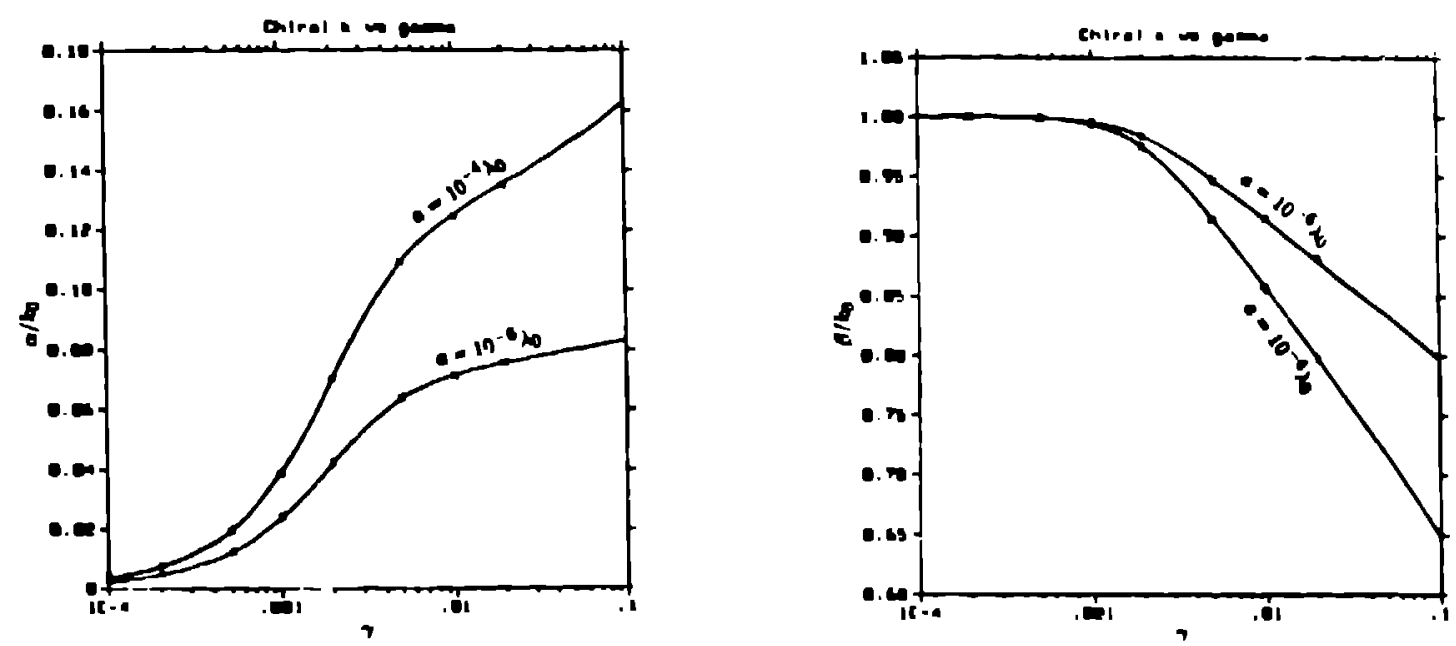

Fig. 1. Comparison of the altenuation $(\alpha)$ and phase $(\beta)$ constants normalized $10 \mathrm{k} 0$ for a wirc ancenna in a chiral medium as a function of chiral parameter $\gamma$ as obtained from NEC (the $x$ 's) and an infinile-antenna model (the solid lines).

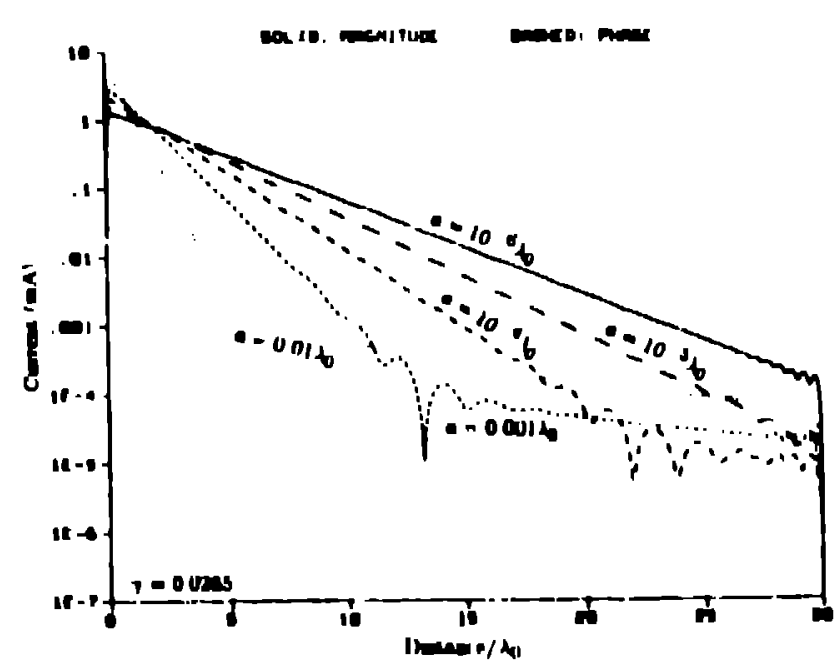

Fig. 2. Current magnitude as a function of distance for 30-wavelength wires of various radii as obluined from NEC for a chiral parneter $\gamma=0.0265$.

Fig. 3. Current magnitude and phase for a wirc of radius $0 .(x)) \lambda_{0}$ for a chiral paramever $\gamma=0.0265$.

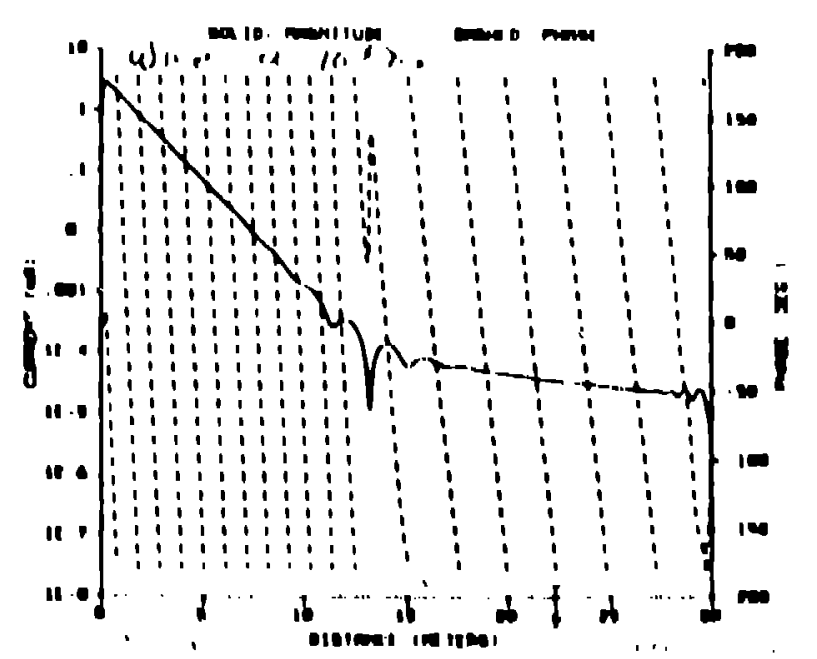

